\title{
EDITORIAL
}

\section{La transformación en la Educación Superior: una mirada de especialización}

\section{y resultados de aprendizaje}

The transformation in Higher Education: a look of specialization and learning outcomes

Filiación:

1. Universidad Santa Paula. Costa

Rica. Director de Docencia.

\section{Correspondencia: $\square$} Jonnathan Enrique GonzálezRodríguez. Correo electónico: jonnathan.gr@gmail.com;

Forma de citar: González Rodríguez JE. La transformación en la Educación Superior: una mirada de especialización y resultados de aprendizaje. Rev Ter. 2018;12(2):4-6.

Financiamiento:

Autofinanciado

Conflictos de Interés:

El autor declara no tener conflictos de interés en la presente publicación
Jonnathan Enrique González-Rodríguez ${ }^{1} \square$

El presente editorial destaca cómo ha cambiado la Educación Superior en las últimas décadas y cuáles son los imperativos que deben configurarla de cara al futuro. Se comenta la relevancia de las Instituciones de Educación Superior como agentes de cambio y promotoras del bienestar, la innovación y el desarrollo humano.

La Educación Superior no puede ni debe ser la misma de las últimas décadas. A todas las personas que poseen una mirada crítica sobre el tema de formación universitaria, les resulta un insumo valioso de análisis el observar la transformación de la sociedad, como producto de los aportes de las Instituciones de Educación Superior (IES), y el cambio que éstas han experimentado como parte de su proceso de armonización con los nuevos desafíos económicos, culturales y científicos que configuran al mundo actual.

No es necesario realizar una comparación exhaustiva entre las características de la Educación Superior de inicios del siglo XIX con las de la actualidad, para reconocer que no sólo enfrentamos un contexto de recursos y proceso educativo distinto, sino también unos retos de futuro críticos para la supervivencia y mejoramiento de las condiciones de vida humana y no humana.

Pese a dichas certezas, hay algo que se ha aferrado a las instituciones educativas con el pasar de los siglos y es la visión de que la transmisión del conocimiento es un asunto de reforzamientos positivos, castigos o creatividad, olvidando por completo la relevancia que tiene el entorno y la autonomía de la persona que aprende para el éxito en los resultados de aprendizaje.

Testigo de lo anterior, son grandes hitos en la historia educativa de Latinoamérica y Europa en los últimos treinta años. En el primer caso, la alta dependencia de los países de la región, en vías de desarrollo, con respecto a modelos clásicos de la educación (permeados del enfoque conductista y repletos de pruebas estándar). En el 
segundo, el declarado fracaso de las acciones ultra constructivistas en la formación de personas que ingresan a la educación terciaria con una escaza o nula habilidad para la creación, sistematización o diseño del conocimiento.

Así entonces, hemos llegado a una época coyuntural, donde la crisis de las metodologías educativas ha puesto sobre la mesa de discusión los conceptos de calidad académica, el rol de la persona docente y el cumplimiento de los perfiles de egreso propuestos por las carreras universitarias. Sin duda alguna, tres elementos que reconfigurarán lo que hoy entendemos como universidad (tanto en sus medios, su forma y los fines que persigue).

Este escenario, no es un pensamiento de pocos, sino que tiende a los consensos de la comunidad académica global. Por ejemplo, la Conferencia Regional de la Educación Superior (CRES) del Instituto Internacional de la Unesco para la Educación Superior en América Latina y el Caribe (IESALC-UNESCO), realizada en CórdobaArgentina, en Julio de 2018 ha proclamado a la Educación Superior como un bien público, con un nuevo significado de calidad: la coherencia entre el quehacer de una IES, su visión estratégica interna y la pertinencia de sus acciones educativas en medio de la realidad social. ${ }^{1,2}$

Esta visión de la CRES, no sólo resulta refrescante, sino novedosa, ya que en los últimos diez años se ha entendido la calidad como acreditación de agencias con procesos de evaluación externa, centrando en un erróneo (la calidad en la ejecución de procesos) como el parámetro de impacto de las IES en el contexto en que se ubican.

En términos de calidad académica, pormenorizando en la enseñanza de las Ciencias de la Salud, es necesario vencer el paradigma de que la mejor enseñanza es predominantemente técnica, hay que priorizar una visión humanística que permita comprender los roles de los equipos interdisciplinarios de salud y sus responsabilidades en la promoción de una sociedad más equitativa, coherente con la defensa de los principio bioéticos más actuales y destacando en áreas como Investigación en Seres Humanos y universalización del acceso a los recursos de salud.

Con respecto al rol de la persona docente, se deben diseñar nuevos métodos que incorporen las herramientas tecnológicas a la dinámica áulica institucional o al espacio clínico, de tal manera que las nuevas actitudes de la persona que estudia sean comprendidas por quien lidera el proceso de enseñanza, sobre todo con el objetivo de hacer que las actividades sean significativas para el futuro profesional y no sólo un trámite en el ínterin de aprobar un plan de estudios.

En el caso del cumplimiento de los perfiles de egreso propuestos, el reto es quizá el más importante: hacer que los planes de estudio sean pertinentes y correspondientes con las necesidades proyectadas de futuro, así como también hacer de la evaluación de los aprendizajes una evaluación del logro de resultados de aprendizaje. Lo anterior implica trascender de una evaluación para verificar conocimientos a una evaluación progresiva para garantizar la adquisición de habilidades profesionales (competencias o atributos de egreso). $3,4,5$

No se puede dejar por fuera de este panorama otros asuntos igual de vinculantes, como lo son la evolución de la virtualidad, la aparición de metodologías postconstructivistas en universidades pioneras en el mundo, el dilema del financiamiento de las IES y la generación de una investigación aplicada que ayude al mejoramiento de las condiciones de vida en el planeta.

Es deber de todas las personas vinculadas al ámbito universitario el asumir su responsabilidad 
en la transformación social, comprender que indistintamente del origen público o privado de las fuentes de Educación Superior, la calidad y la pertinencia no pueden ser supeditadas a un segundo plano. Asimismo, y en referencia a lo que plantea Immanuel Kant plantea su imperativo categórico, hoy estamos llamados a resignificar a la universidad no como un corporativo con fines industriales o mercadotécnicos, sino más bien como un centro de especialización garante de la innovación y los Derechos Humanos.

\section{Referencias}

1. Grimaldo H, coordinador. El rol de la educación superior de cara a los desafíos sociales en América Latina y el Caribe. En: Conferencia Regional de Educación Superior; 2018; Córdoba. Córdoba, AR: UNESCO, IESALC, Universidad Nacional de Córdoba; 2018.

2. Henríquez Guajardo P, coordinador. El papel estratégico de la educación superior en el desarrollo de sostenible de América Latina y el Caribe. En: Conferencia Regional de Educación Superior; 2018; Córdoba. Córdoba, AR: UNESCO, IESALC, Universidad Nacional de Córdoba; 2018.

3. Agencia de Calidad de la Educación [Internet]. Santiago, CL: Agencia de Calidad de la Educación; 2018 [citado el 30 de noviembre de 2018]. Evaluación progresiva. Disponible en: https://www.agenciaeducacion.cl/evaluaciones/evaluacion-progresiva/.

4. Alvarado M, Audibert M, Cifuentes C, Gómez M, Mella E, Román A. Hitos evaluativos para evidenciar el logro de los perfiles. En: Centro Interuniversitario de Desarrollo. Evaluación del logro de perfiles de egreso: Experiencias universitarias. Santiago, CL: CINDA; 2017. 65-108.

5. Ampuero N, Báez M, Castillo C, Lagos J, Loncomilla L, Núñez $F$ et al. La evaluación progresiva del logro del perfil del egreso. En: Centro Interuniversitario de Desarrollo. Evaluación del logro de perfiles de egreso: Experiencias universitarias. Santiago, CL: CINDA; 2017. 111-119. 